\title{
Li K-Emission Measurements Using a Newly Developed SXES-TEM Instrument
}

\author{
M. Terauchi ${ }^{1}$, H. Takahashi ${ }^{2}$, N. Handa ${ }^{3}$, T. Murano ${ }^{3}$, M. Koike ${ }^{4}$, T. Kawachi ${ }^{4}$, T. Imazono ${ }^{4}$, \\ M. Koeda ${ }^{5}$, T. Nagano ${ }^{5}$, H. Sasai ${ }^{5}$, Y. Oue ${ }^{5}$, Z. Yonezawa ${ }^{5}$ and S. Kuramoto ${ }^{5}$ \\ ${ }^{1}$ IMRAM, Tohoku University, 2-1-1 Katahira, Aoba-ku, Sendai 980-8577, Japan. \\ 2 JEOL Ltd., 3-1-2 Musashino, Akishima, Tokyo 196-8558, Japan. \\ ${ }^{3}$ JEOL Engineering Co. Ltd., 3-1-2 Musashino, Akishima, Tokyo 196-8558, Japan. \\ ${ }^{4}$ APRC, Quantum Beam Science Directorate, Japan Atomic Energy Agency, 8-1 Umemidai, \\ Kizugawa, Kyoto 619-0215, Japan. \\ ${ }^{5}$ Consumer \& Optical Products Department, SHIMADZU Corp., 1 Nishinokyo-Kuwabaracho, \\ Nakagyo-ku, Kyoto 604-8511, Japan.
}

Soft X-ray emission spectroscopy (SXES) gives information of electronic structure of bonding electrons. Then, SXES combined with microscopy should be a hopeful method to reveal physical properties and chemical bonding states of identified small specimen areas of various compounds. Terauchi et al. reported two types of SXES instruments for conventional transmission electron microscopes [1]. The spectrometers basically designed to detect from $60 \mathrm{eV}$ to $1200 \mathrm{eV}$. Extended version can detect more than $2000 \mathrm{eV}$ [2]. For applying to material science, a much wider energy range is necessary. Thus, a new SXES development for electron microscope has started to obtain an energy range from $50 \mathrm{eV}$ to $3800 \mathrm{eV}$.

Figure 1 shows a photo of a newly constructed SXES spectrometer attached to a transmission electron microscope (TEM) of JEM2010. This spectrometer can set four gratings. Two types of detectors are attached. One detector (lower detector in Fig.1) for higher energy region is a back-thin-type CCD with a pixel size of $12 \mu \mathrm{m}$. Another one (upper detector in Fig.1) is especially for lower energy region. It is a conventional front-illumination-type CCD optically coupled with a multi-channel plate (MCP). This detector has a larger pixel size of about $24 \mu \mathrm{m}$ causing a lower energy resolution compared with that of CCD. However, it does not cause a serious drawback, because large energy dispersion is expected in a low energy region (long-wavelength region). An additional important function of MCP is to reduce background intensity due to luminescence in visible light energy region from specimens. Those detectors can change keeping a high vacuum condition. At present, a new grating JS-50XL for 50-200eV has installed. An energy resolution of $0.3 \mathrm{eV}$ was confirmed by a sharp Fermi edge for $\mathrm{Al}$ L-emission.

Figure 2 shows Li K-emission obtained by using the new grating and the MCP+CCD detector. Since there was not observed O K-emission intensity, the spectrum corresponds to 
metal Li. The energy position of Li-K peak was adjusted for a reported value of $54.7 \mathrm{eV}$ [3]. Steep intensity change in right had side of $\mathrm{Li}-\mathrm{K}$ spectrum should correspond to Fermi edge. From the sharpness of the edge is $0.3 \mathrm{eV}$, which is the same with the energy resolution. Since emission efficiency is extremely low in low-energy region, for example $7.5 \times 10^{-4}$ for $\mathrm{Al} \mathrm{L-emission,} \mathrm{this}$ grating works much better in high beam-current microscope like an electron-probe microanalyzer (EPMA).

This development is conducting as one project of Collaborative Development of Innovative Seeds (Practicability verification stage) by Japan Science and Technology Agency.

References [1] M.Terauchi et al.: Proc. of 16th Int. Conf. Microscopy, 892 (2006). [2] M.Terauchi et al.: submitted to J. Electron Microscopy (2010). [3] J.A.Bearden: Review of Modern Physics, vol.39, 78 (1969).
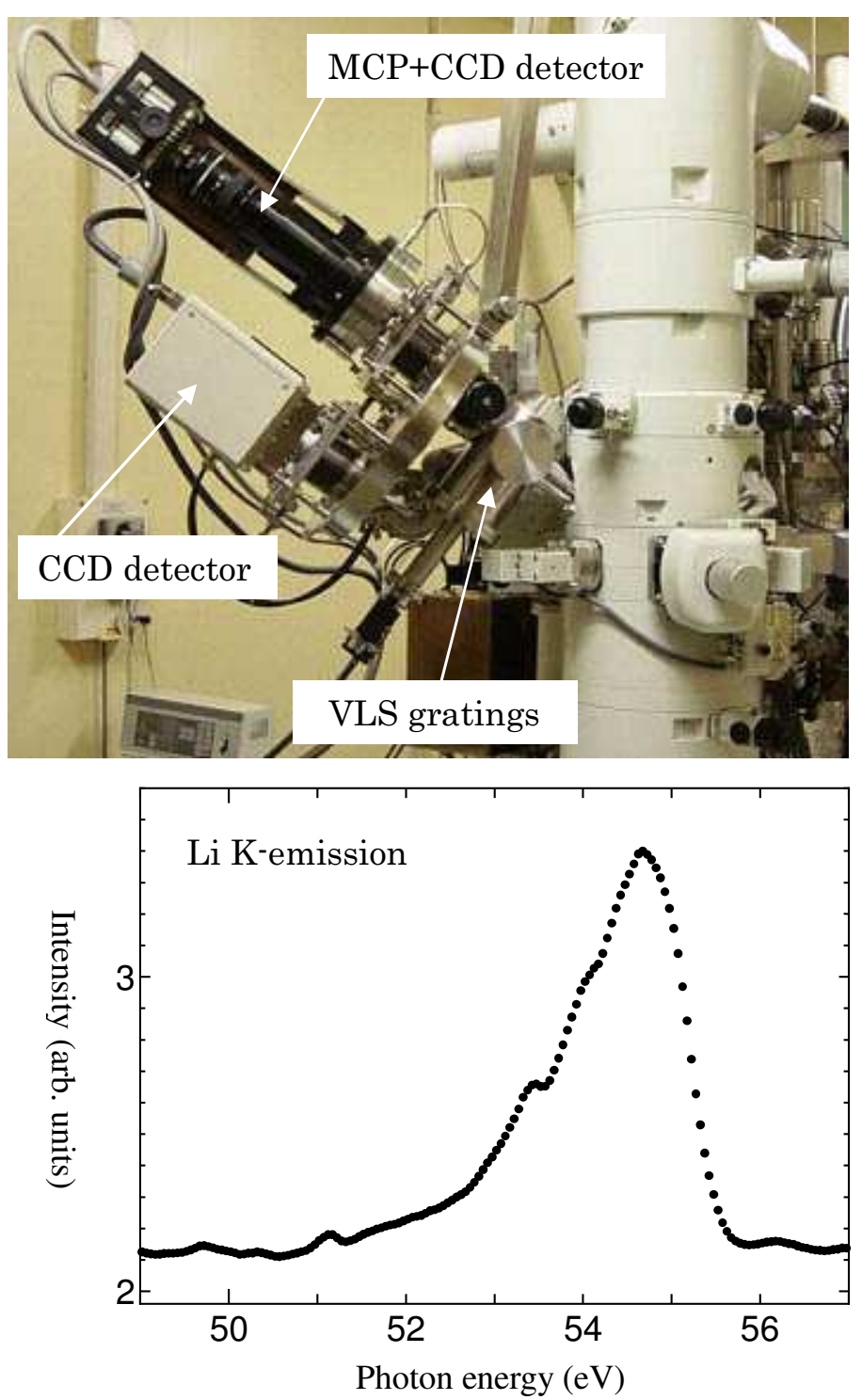

FIG 1

Newly constructed SXES-TEM instrument. Four gratings can be introduced. Two different types of detectors are attached. Upper one $(\mathrm{MCP}+\mathrm{CCD})$ is for low energy region. MCP effectively reduces background intensity due to luminescence in visible energy region from specimen. Pixel size is 24 $\mu \mathrm{m}$. Lower one (CCD) is for higher energy resolution. Pixel size is $12 \mu \mathrm{m}$. Those detectors can change keeping a high vacuum condition.

FIG 2

Li K-emission from metallic Li obtained by the $\mathrm{MCP}+\mathrm{CCD}$ detector. A sharp intensity change in the right hand side corresponds to Fermi edge, which shows a sharpness of $0.3 \mathrm{eV}$ of the energy resolution of the detector. 